\title{
Lower Omega-3 Index Is a Marker of Increased Propensity of Hypertensive Rat Heart to Malignant Arrhythmias
}

\author{
B. BAČOVÁ ${ }^{1^{*}}$, P. SEČ ${ }^{2 *}$, J. RADOŠINSKÁ ${ }^{3}$, M. ČERTÍK ${ }^{4}$, A. VACHULOVÁ ${ }^{5}$ \\ N. TRIBULOVÁ ${ }^{\mathbf{1}}$ \\ * These authors contributed equally to this work.
}

${ }^{1}$ Institute for Heart Research, Slovak Academy of Sciences, Bratislava, Slovakia, ${ }^{2}$ Institute of Biochemistry and Genetic of Animals, Slovak Academy of Sciences, Bratislava, Slovakia, ${ }^{3}$ Institute of Physiology, Medical Faculty of Comenius University, Bratislava, Slovakia, ${ }^{4}$ Faculty of Chemical and Food Technology, Slovak University of Technology, Bratislava, Slovakia, ${ }^{5}$ Department of Arrhythmias, National Cardiovascular Institute, Bratislava, Slovakia

Received March 14, 2013

Accepted July 29, 2013

\begin{abstract}
Summary
Polyunsaturated omega-3 fatty acids ( $\omega$-3 PUFA) are important components of cell membrane affecting its function and their deficiency is deleterious to health. We have previously shown that spontaneously hypertensive rats (SHR) are prone to lifethreatening arrhythmias that are reduced by $\omega-3$ PUFA intake. Purpose of this study was to explore plasma and red blood cells (RBC) profile of $\omega-3$ and $\omega-6$ PUFA as well as to determine $\omega-3$ index, a risk factor for sudden cardiac death, in aged SHR and the effect of $\omega-3$ PUFA intake. Male and female 12-month-old SHR and age-matched Wistar rats fed with $\omega-3$ PUFA $(200 \mathrm{mg} / \mathrm{kg}$ BW/day/2 month) were compared with untreated rats. Composition of $\omega-3$ PUFA: alfa linolenic acid, eicosapentaenoic acid (EPA) and docosahexaenoic acid (DHA) as well as $\omega-6$ PUFA: linoleic acid and arachidonic acid was analyzed by gas chromatography. Results showed sex- and strain-related differences of basal $\omega-3$ and $\omega-6$ PUFA levels in plasma and RBC as well as in response to $\omega-3$ PUFA intake. Comparing to Wistar rats $\omega-3$ index, expressed as a percentage of EPA+DHA of total fatty acids, was lower in SHR and it increased due to consumption of $\omega-3$ PUFA. Findings support our hypothesis that lower $\omega-3$ index may be also a marker of increased propensity of the hypertensive rat heart to malignant arrhythmias.
\end{abstract}

\section{Key words}

SHR $\bullet \omega-3$ index $\bullet \omega-3$ PUFA intake

\section{Corresponding author}

Narcis Tribulová, Institute for Heart Research, Slovak Academy of Sciences, 84005 Bratislava, Dúbravská cesta 9, POBox 104, Fax: 00421254776637. E-mail: narcisa.tribulova@savba.sk

\section{Introduction}

Sudden cardiac death (SCD) accounts for more than $15 \%$ of all deaths in Europe, and therefore is a major health problem (Zippes et al. 2006). SCD is the major cause of death due to ventricular tachyarrhythmias, particularly ventricular fibrillation (VF). It may be caused by various arrhythmogenic factors. One of these factors is myocardial hypertrophy. Ventricular hypertrophy is characterized by several electro-physiological abnormalities including prolonged duration of the action potential, decreased resting membrane potential, heterogeneous recovery following depolarization and slowed conduction velocity (Zipes et al. 2006). The latter can be attributed to interstitial fibrosis and gap junction connexin-43 channels remodeling and/or down-regulation (Mitasikova et al. 2008, Radosinska et al. 2013). All of these abnormalities as well as altered intracellular $\mathrm{Ca}^{2+}$ handling may facilitate the genesis and maintenance of malignant ventricular arrhythmias (Tribulova et al. 2009). Attempts to improve prevention of SCD have focused on implantable automated defibrillators (ICDs). However, ICDs are only implanted in a minority of patients fulfilling guidelines criteria (Epstein et al. 2008). Moreover, ICD does not prevent VF occurrence. Thus, other approaches need to be explored to prevent SCD. 
The most promising approach seems to be use of the two marine omega-3 polyunsaturated fatty acids ( $\omega-3$ PUFA): eicosapentaenoic acid (EPA) and docosahexaenoic acid (DHA) (von Schacky 2010).

Data from clinical (London et al. 2007, Rupp et al. 2012) and experimental studies (Mitasikova et al. 2008, Nair et al. 1997) support the hypothesis that consumption of $\omega-3$ PUFA lowers the risk of cardiovascular diseases and SCD (Rupp et al. 2012, von Schacky and Harris 2007). Based on the scientific evidence it was proposed a new risk factor for SCD, the w-3 index (von Schacky and Harris 2007). It is measured in red blood cells (RBC) and is expressed as a percentage of EPA+DHA of total fatty acids. RBC fatty acid composition reflects long-term intake of EPA and DHA as well as tissue fatty acids composition, including the cardiac muscle (Harris and von Schacky 2004). Polyunsaturated $\omega-3$ fatty acids are important components of cell membranes affecting their function, as they are incorporated into the phospholipids. Dietary deprivation of the essential fatty acids is deleterious to health. We have previously shown that $\omega$-3 PUFA intake protects young and old spontaneously hypertensive rats (SHR) from VF (Mitasikova et al. 2008, Radosinska et al. 2011, Radosinska et al. 2013). Protection was associated with treatment-induced improvement of cell membrane and intercellular junction integrity as well as up-regulation of myocardial electrical coupling protein, connexin-43. This study was aimed to explore plasma and RBC profile of $\omega-3$ and $\omega-6$ PUFA as well as to determine $\omega-3$ ndex in aged male and female SHR and the effect of $\omega-3$ PUFA intake.

\section{Methods}

All animal experiments were performed in accordance with the rules issued by the State Veterinary Administration of the Slovak Republic, legislation No 289/2003 and they conform to the 'European Convention for the Protection of Vertebrate Animals used for Experimental and other Scientific Purposes' (Council of Europe No 123, Strasbourg 1985). Animals were purchased from Dobra Voda (Slovakia). Experiments were conducted on male and female, 12-month-old SHR as well as their age-matched healthy Wistar rats. Animals were divided into groups of untreated male and female rats $(n=40)$ fed by standard laboratory chow and treated rats $(n=40)$ supplemented orally with $\omega-3$ ethyl-ester (Vesteralens, Norway product Omega-3E containing
$105 \mathrm{mg}$ DHA, $160 \mathrm{mg}$ EPA, other $\omega-3$ fatty acids $45 \mathrm{mg}$, natural vitamin E $15 \mathrm{mg}$ ) in dose $200 \mathrm{mg} / \mathrm{kg} \mathrm{BW} /$ day for two month as previously described (Radosinska et al. 2011). The animals were anesthetized with thiopental $(50 \mathrm{mg} / \mathrm{kg}$, ip), the chest was open and blood was collected from heart.

\section{Animal monitoring and tissue sampling}

Systolic blood pressure (sBP) was measured by tail-cuff plethysmography using the Statham Pressure Transducer P23XL (Hugo Sachs, Germany) and monitored together with body weight (BW) at the beginning and the end of the experiments. The hearts from the anaesthetized rats were rapidly excised into icecold saline to arrest heart beat and the weight of the heart (HW) and left ventricle (LVW) was registered. Blood from the heart was drawn into ethylenediaminetetraacetic acid tubes and centrifuged at $4{ }^{\circ} \mathrm{C}$ to separate cells from plasma. The buff coat was removed and plasma and $\mathrm{RBC}$ were frozen in liquid nitrogen and kept at $-80{ }^{\circ} \mathrm{C}$ until analyzed for fatty acids composition by gas chromatography.

\section{Lipid extraction and fatty acids analysis}

Total lipids from plasma and red blood cells samples were extracted three times with chloroform containing internal standard of heptadecaenoic acid (Supelco, USA). Collected chloroform layers were evaporated under vacuum and lipids were dissolved in hexane/chloroform $(9: 1, \mathrm{v} / \mathrm{v})$. Fatty acids from total lipids were converted to their methyl-esters by methanolic solution of sodium methoxide and methanolic $\mathrm{HCl}$ and analyzed by gas chromatography (GC-6890 N, Agilent Technologies) using a capillary column DB-23 and a FID detector as previously described (Sukrutha et al. 2012). The fatty acid methyl-ester peaks were identified by authentic standards of $\mathrm{C}_{4}-\mathrm{C}_{24}$ fatty acid methyl-esters mixture (Supelco, USA) and evaluated by ChemStation $\mathrm{B}$ 0103 (Agilent Technologies). To identify the unknown peaks in lipid structures, GC-MS analysis of fatty acid methyl-esters was also performed. Detection limit was 0.1 microgram of fatty acid methyl-ester. All peaks were evaluated by MSD ChemStation E.00.00.202 with combination of NIST Mass Spectral Search Program version $2.0 \mathrm{~d}$ (Agilent Technologies). The levels of major $\omega$-3 PUFA, i.e. ALA (alfa linolenic acid), EPA and DHA as well as $\omega-6$ PUFA, i.e. LA (linoleic acid) and AA (arachidonic acid) was expressed in percentage of total free fatty acids. $\omega-3$ index was expressed as a percentage 
Table 1. Characteristics of male and female Wistar and SHR.

\begin{tabular}{|c|c|c|c|c|}
\hline Male & WRe & $\mathbf{W R}+\omega 3$ & SHRc & $\mathrm{SHR}+\omega 3$ \\
\hline$s B P(m m H g)$ & $121 \pm 7$ & $90 \pm 4 * \#$ & $183 \pm 11^{*}$ & $178 \pm 7$ \\
\hline$B W(g)$ & $465 \pm 37$ & $432 \pm 18 * \#$ & $337 \pm 6^{*}$ & $333 \pm 21$ \\
\hline$B W$ gain $(g)$ & 73.3 & 57.7 & 18.1 & 1.1 \\
\hline$H W(g)$ & $1.10 \pm 0.09$ & $1.06 \pm 0.05$ & $1.48 \pm 0.16^{*}$ & $1.39 \pm 0.08$ \\
\hline$L V W(g)$ & $0.80 \pm 0.04$ & $0.82 \pm 0.04$ & $1.26 \pm 0.25^{*}$ & $1.15 \pm 0.04$ \\
\hline$H W / B W(m g / g)$ & $2.36 \pm 0.20$ & $2.50 \pm 0.08$ & $4.30 \pm 0.12 *$ & $4.17 \pm 0.20$ \\
\hline Female & WRe & $\mathbf{W R}+\omega 3$ & SHRc & $\mathrm{SHR}+\omega 3$ \\
\hline$s B P(m m H g)$ & $106 \pm 3$ & $95 \pm 5 * \#$ & $189 \pm 14^{*}$ & $159 \pm 18 \#$ \\
\hline$B W(g)$ & $264 \pm 22$ & $294 \pm 30 * \#$ & $211 \pm 15^{*}$ & $236 \pm 17 \#$ \\
\hline$B W$ gain (g) & 4.1 & 30.3 & 1.3 & 2.4 \\
\hline$H W(g)$ & $0.89 \pm 0.06$ & $0.85 \pm 0.09$ & $1.42 \pm 0.17^{*}$ & $1.26 \pm 0.15 \#$ \\
\hline$L V W(g)$ & $0.70 \pm 0.05$ & $0.68 \pm 0.07$ & $1.10 \pm 0.20 *$ & $0.88 \pm 0.15 \#$ \\
\hline$H W / B W(m g / g)$ & $3.37 \pm 0.30$ & $2.89 \pm 0.29 * \#$ & $6.72 \pm 1.08 *$ & $5.34 \pm 0.59 \#$ \\
\hline
\end{tabular}

Values are means \pm SD of 10 rats in each group. WRc - Wistar control rats, WR+ $\omega 3-$ Wistar rats fed with $\omega-3$ fatty acid, SHRc - SHR control rats, SHR $+\omega 3$ - SHR rats fed with $\omega-3$ fatty acid, sBP - systolic blood pressure, BW - body weight, HW - heart weight, LVW left ventricular weight. Significant difference from WRc: $* p<0.05$. Significant difference between rats supplemented by $\omega-3$ PUFA: \# $\mathrm{p}<0.05$.

of EPA+DHA of total fatty acids measured in RBC.

\section{Statistical analysis}

The data are expressed as means \pm standard deviations (SD). One-way ANOVA followed by Newmann-Keuls test was used to analyze the statistical significance between means. Comparison between two groups was performed using the two-tailed Student's ttest. Values were considered to differ significantly at $\mathrm{p}<0.05$.

\section{Results}

\section{Main characteristics of experimental rats}

Both male and female hypertensive rats exhibited higher sBP as well as HW and LVW compared to non-hypertensive rats. BW of male and female SHR was lower comparing to age-matched Wistar rats. Consumption of $\omega-3$ PUFA reduced significantly sBP in female but not in male SHR. Moreover, $\omega-3$ PUFA supplementation reduced HW and LVW in male and significantly in female SHR. Furthermore, it decreased $\mathrm{BW}$ in male Wistar rats but not SHR and increased BW in females of both strains. Data are summarized in Table 1.

\section{Fatty acids levels in plasma}

Comparing to Wistar rats the circulating levels of EPA and DHA in plasma were lower in male and to lesser extent in female SHR as well. ALA was not detected in males either Wistar or SHR and its content was very low (about $0.03 \%$ ) in females of both strains. On the other hand, LA levels were higher than $0.3 \%$ whereby there was no apparent difference between males Wistar and SHR. However, in females LA was lower in SHR comparing to Wistar rats. Intake of $\omega-3$ PUFA resulted in significant increase of EPA but not DHA in plasma of Wistar and SHR regardless the sex. Moreover, consumption of $\omega-3$ PUFA led to a significant increase of LA in male and female SHR as well as Wistar rats. Data are presented in Table 2 .

\section{Fatty acids levels in red blood cells}

Comparing to Wistar rats RBC levels of EPA and particularly DHA but not ALA were lower in male and female SHR (Fig. 1AB). Interestingly, AA was almost six times higher in males than females either SHR or Wistar rats (Fig. 2AB). On the other hand, LA level was lower in male but higher in female SHR compared to Wistar rats (Fig. 2AB). Intake of $\omega-3$ PUFA resulted in significant increase EPA and DHA in SHR and Wistar rats regardless the sex (Fig. 1AB). The increase of EPA 
Table 2. Plasma $\omega-6$ PUFA and $\omega-3$ PUFA levels expressed in $\%$ of total fatty acids in male and female Wistar rats and SHR.

\begin{tabular}{|c|c|c|c|c|}
\hline Male & WRe & $\mathrm{WR}+\omega 3$ & SHRc & $\mathrm{SHR}+\omega 3$ \\
\hline$L A$ & $0.30 \pm 0.04$ & $0.45 \pm 0.07 * \#$ & $0.29 \pm 0.07$ & $1.20 \pm 0.31 \#$ \\
\hline$A A$ & $0.03 \pm 0.004$ & $0.08 \pm 0.02 * \#$ & $0.11 \pm 0.03^{*}$ & $0.14 \pm 0.04$ \\
\hline$E P A$ & $0.15 \pm 0.05$ & $0.71 \pm 0.07 * \#$ & $0.02 \pm 0.002 *$ & $0.28 \pm 0.08 \#$ \\
\hline$D H A$ & $0.33 \pm 0.08$ & $0.28 \pm 0.09$ & $0.06 \pm 0.04 *$ & $0.07 \pm 0.01$ \\
\hline Female & WRe & $\mathrm{WR}+\omega 3$ & SHRc & $\mathrm{SHR}+\omega 3$ \\
\hline$L A$ & $0.8 \pm 0.1$ & $1.7 \pm 0.05^{* \#}$ & $0.31 \pm 0.02 *$ & $0.45 \pm 0.06 \#$ \\
\hline$A A$ & $0.03 \pm 0.004$ & $0.08 \pm 0.02 * \#$ & $0.08 \pm 0.003 *$ & $0.10 \pm 0.01 \#$ \\
\hline$E P A$ & $0.02 \pm 0.01$ & $0.5 \pm 0.03^{* \#}$ & $0.06 \pm 0.02 *$ & $0.2 \pm 0.03 \#$ \\
\hline DHA & $0.07 \pm 0.01$ & $0.06 \pm 0.02$ & $0.06 \pm 0.01 *$ & $0.05 \pm 0.01 \#$ \\
\hline$A L A$ & $0.03 \pm 0.004$ & $0.04 \pm 0.002 * \#$ & $0.03 \pm 0.002$ & $0.03 \pm 0.001$ \\
\hline
\end{tabular}

LA - linoleic acid, AA - arachidonic acid, EPA - eicosapentaenoic acid, DHA - docosahexaenoic acid, ALA - alfa linolenic acid. Values are means $\pm S D$ of 10 rats in each group. Significant difference from untreated rats: $* p<0.05$. Significant difference between rats supplemented by $\omega-3$ PUFA: $\# p<0.05$.

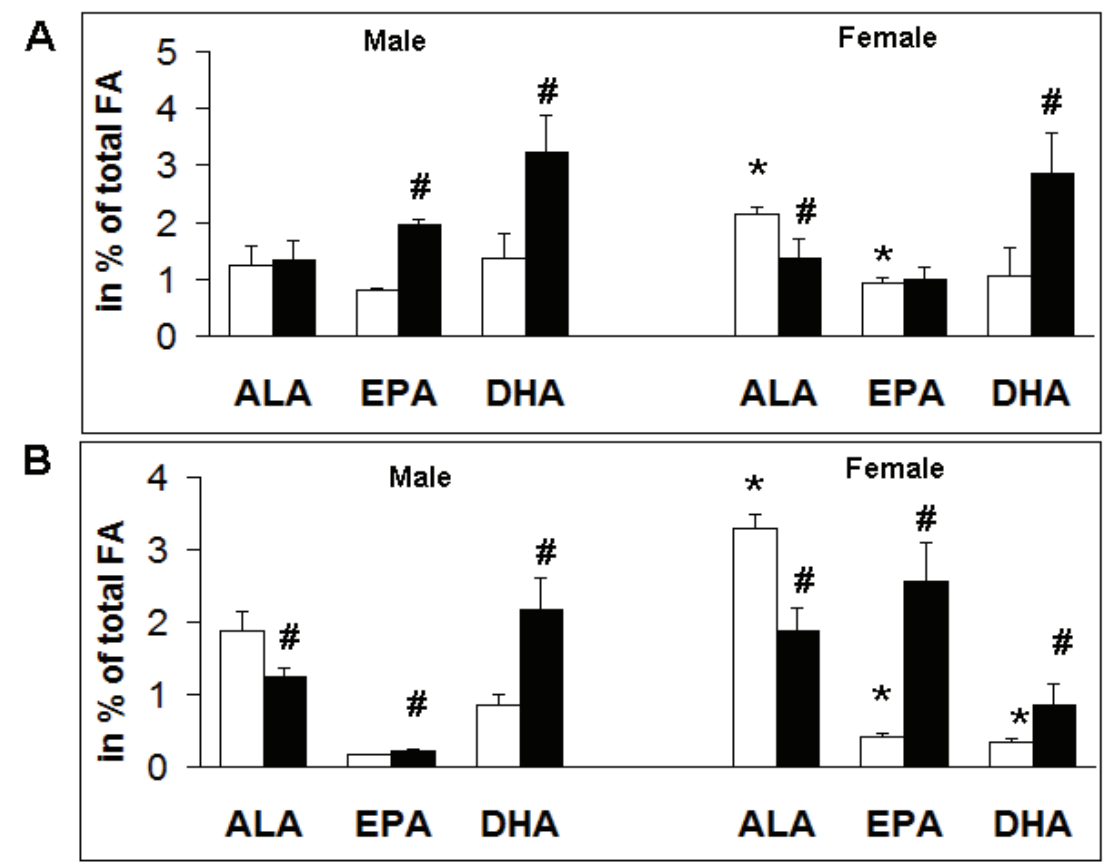

Fig. 1. Red blood cell $\omega-3$ PUFA levels in male as well as female Wistar (A) and SHR (B). ALA - alfa linolenic acid, EPA eicosapentaenoic acid, DHA docosahexaenoic acid; untreated rats white columns; $\omega-3$ PUFA-treated rats black columns. Values are means \pm SD of 10 rats in each group. Significant difference versus male and female: $* \mathrm{p}<0.05$. Significant difference versus treated and untreated rats: $\# p<0.05$. in female Wistar rats was, however, not significant. Feeding of rats with $\omega-3$ PUFA did not affect significantly LA and AA in males either SHR or Wistar rats but increased LA in female Wistar rats and to lesser extent in SHR as well as AA in female SHR (Fig. 2AB). Total $\omega-6$ PUFA levels were higher in males comparing to females either Wistar or SHR. On the other hand, there was no difference in total $\omega$-3 PUFA levels between Wistar and SHR regardless the sex. $\omega-3$ index, i.e. EPA+DHA was lower in both male and female SHR comparing to age- matched Wistar rats. This parameter was, however, increased due to $\omega-3$ PUFA intake in both strains regardless the sex. Moreover, treatment was associated with a decrease in AA/EPA ratio in male as well as female SHR and Wistar rats. Data are summarized in Table 3.

\section{Discussion}

To our knowledge we have determined for the first time $\omega-3$ and $\omega-6$ PUFA profile in plasma and RBC 


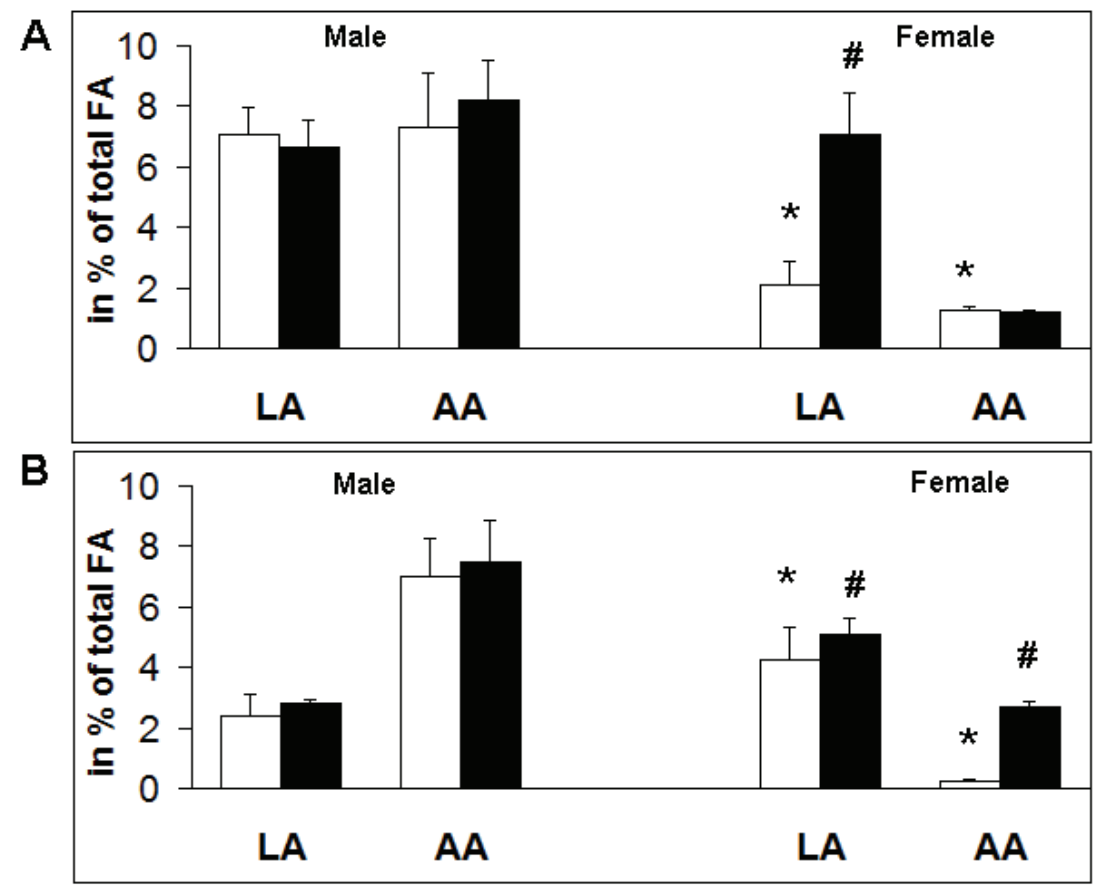

Fig. 2. Red blod cell $\omega-6$ PUFA levels in male as well as female Wistar (A) and SHR (B). LA - linoleic acid, AA - arachidonic acid; untreated rats - white columns; $\omega-3$ PUFA-treated rats - black columns. Values are means \pm SD of 10 rats in each group. Significant difference versus male and female: $* p<0.05$. Significant difference versus treated and untreated rats: $\# \mathrm{p}<0.05$.

of aged healthy and hypertensive rats. We have found that there are strain- and sex-dependent differences in $\omega-3$ and $\omega-6$ PUFA levels in both plasma and RBC. More importantly, $\omega-3$ index, i.e. EPA + DHA was lower in SHR when compared to Wistar rats and it was enhanced due to $\omega-3$ PUFA supplementation. In addition, $\omega-3$ PUFA intake resulted in decrease of sBP, HW and LVW in female SHR.

It should be noted that $\omega-3$ and $\omega-6$ PUFA profile found in plasma reflects short-term, whereas in RBC rather long-term changes in dietary fatty acids intake. Comparing to males the plasma $\omega-3$ but not $\omega-6$ PUFA content was lower in females either Wistar or SHR. It may indicate that the rate of $\omega-3$ PUFA metabolism is faster in females even if they are old. Women also have a greater capacity than man to synthesize $\omega$-3 PUFA from their essential fatty acid precursor ALA (Childs et al. 2012). Moreover, turnover of $\omega-3$ PUFA in plasma of old males decreased with age (Gao et al. 2013). The similar feature of plasma profile of PUFA as revealed in male Wistar rats (including higher concentration of LA) has been found by others in young or middle-age healthy male rats (Tranchida et al. 2012, Taha et al. 2012, Zeghichi-Hamri et al. 2010). However, the data in old animals are missing. Interestingly, likewise healthy humans (Viviani Anselmi et al. 2010) the levels of DHA in plasma or RBC were higher than EPA in Wistar rats but not in SHR. The content of ALA in RBC was apparently higher than EPA or DHA in all rats examined. Thus, it is most likely that ALA is
Table 3. Red blood cell levels of registered $\omega-6$ PUFA as well as $\omega-3$ PUFA and omega-3 index in male and female Wistar and SHR.

\begin{tabular}{|c|c|c|c|c|}
\hline Male & WRe & $\mathbf{W R}+\omega 3$ & SHRc & $\mathrm{SHR}+\omega 3$ \\
\hline$\omega-6 F A(\%)$ & 14.4 & 13.5 & 8.5 & 10.5 \\
\hline$\omega-3 F A(\%)$ & 3.4 & 6.5 & 2.9 & 3.9 \\
\hline$\omega-3$ index & 2.2 & 5.2 & 1 & 2.4 \\
\hline$A A / E P A$ & 8.4 & 4.2 & 37.3 & 34.1 \\
\hline Female & WRe & $\mathbf{W R}+\omega 3$ & SHRc & $\mathrm{SHR}+\omega 3$ \\
\hline$\omega-6 F A(\%)$ & 3.4 & 8.2 & 4.5 & 7.8 \\
\hline$\omega-3 F A(\%)$ & 4.1 & 5.1 & 4 & 5.3 \\
\hline$\omega-3$ index & 2 & 3.7 & 0.7 & 3.4 \\
\hline$A A / E P A$ & 1.4 & 1.3 & 1.6 & 1 \\
\hline
\end{tabular}

WRc - Wistar control rats, WR+ $\omega 3$ - Wistar rats fed with $\omega-3$ PUFA, SHRC - SHR control rats, SHR+ $\omega 3$ - SHR rats fed with $\omega-3$ PUFA

dominant $\omega$-3 PUFA in tissue cell membranes of old rats. Of note, ALA was higher in females than males regardless the strain. In contrast, $\mathrm{RBC}$ levels of AA (important representative of $\omega-6$ PUFA) were much lower in females than males SHR as well as Wistar rats. Furthermore, LA (precursor of $\omega-6$ PUFA) was low in female Wistar but not SHR. Our findings also suggest that male rats exhibit higher level of $\omega-6$ PUFA comparing to $\omega-3$ PUFA. Sex-related differences point 
out the role of sex hormones in regulation of PUFA metabolism. It has been hypothesized that sex differences are established in order to ensure adequate supply of PUFA to the developing fetus (Childs et al. 2010). Different to ALA (which does not exhibit strain differences) the levels of EPA or DHA were lower in SHR comparing to Wistar rats regardless the sex. Consequently, $\omega-3$ index was lower in SHR than Wistar rats. Accordingly, it appears that low levels of EPA and DHA are associated with increased propensity to lifethreatening arrhythmias demonstrated previously in old SHR (Mitasikova et al. 2008, Radosinska et al. 2013).

The main results of our study point out that two month-lasting supplementation with $\omega$-3 PUFA ethylester resulted in significant increase of both plasma and RBC levels of EPA and/or DHA in Wistar and particularly SHR. An increase of EPA and DHA was accompanied by reduction on ALA levels perhaps due to its enhanced metabolism and conversion to EPA or DHA. Moreover, there was apparent strain- and sex-dependent difference in treatment response to RBC fatty acids profile. Increase of EPA in males was higher in Wistar rats comparing to SHR while this relationship was opposite in females. Noteworthy, AA was not changed due to $\omega-3$ PUFA intake in RBC of males unlike the females in which its level was significantly increased in SHR but suppressed in Wistar rats (decrease was also shown by Zeghichi-Hamri et al. 2010). Interestingly, $\omega-3$ PUFA-induced increase of AA was associated with significant decrease of the blood pressure in females SHR. However, blood pressure was not affected in male SHR in which AA did not change. It may indicate implication of AA in modulation of vascular resistance. In this context it should be noted the dual role of AA in biological processes. Not all eicosanoids derived from AA are pro-inflammatory or adverse for vascular function. Indeed, AA stimulates production of prostacyclin $\left(\mathrm{PGI}_{3}\right)$ by vascular tissue, a compound that exhibit antithrombotic effects and intake of $\omega-3$ PUFA enhanced it (DeCaterina et al. 1990). Moreover, AA is converted to epoxyeicosatrienoic acids (EETs) by epoxygenases or to 20-hydroxyeicosatetraenoic acid (20HETE) by omega-hydrolases (Yousif and Benter 2010). 20-HETE, a vasoconstrictor associated with endothelial dysfunction, is higher in hypertensive individuals as well as SHR and androgens increase its synthesis (Pearson et al. 2009). Thus, it appears that males might be more prone to hypertension than females. On the other hand, EETs are vasodilators and anti-inflammatory mediators. They are antihypertensive, since their intravenous infusion significantly reduced blood pressure in SHR (Lin et al. 1990). RBC are reservoirs and carriers of EETs in the circulation, thereby in addition to $\mathrm{O}_{2}$ delivery they participate in the regulation of microvascular tone by releasing vasoactive factors, including EETs (Jiang et al. 2011). Importantly, AA/EPA ratio was higher in SHR than Wistar rats while decreased due to $\omega-3$ PUFA intake. Of note, reduced ratio AA/EPA shifts the spectrum of ecosanoids production toward increase in tromboxane $\mathrm{A}_{3}$ and $\mathrm{PGI}_{3}$ at the expense of $\mathrm{TXA}_{2}$ and $\mathrm{PGI}_{2}$. This shift was found to reduce risk of fatal arrhythmias (Coker et al. 1982).

In conclusion, this study revealed for the first time that $\omega-3$ index is lower in male and female SHR comparing to Wistar rats but increased due to $\omega-3$ PUFA intake. It should be emphasized the inverse association of $\omega-3$ index with occurrence of malignant arrhythmias in SHR. Findings support our hypothesis that lower $\omega-3$ index may be a marker of increased propensity of the heart to SCD due to malignant arrhytmia.

\section{Conflict of Interest}

There is no conflict of interest.

\section{Acknowledgements}

This study was realized by support of VEGA 2/0046/12, 1/0975/12, APVV-SK-CZ-0027-11, APVV-0662-11 and SKS grants. Omega-3 fatty acids ethyl-esters were generous gift of Vesteralens Company, Norway.

\section{References}

CHILDS CE, ROMIJN T, ENKE U, HOILE S, CALDER PC: Maternal diet during pregnancy has tissue-specific effects upon fetal fatty acid composition and alters fetal immune parameters. PLEFA 83: 179-184, 2010.

CHILDS CE, HOILE SP, BURDGE GC, CALDER PC: Changes in rat $\mathrm{n}-3$ and $\mathrm{n}-6$ fatty acid composition during pregnancy are associated with progesterone concentrations and hepatic FADS2 expression. PLEFA 86: 141$147,2012$. 
COKER SJ, PARRATT JR, LEDINGHAM IM, ZEITLIN IJ: Evidence that thromboxane contributes to ventricular fibrillation induced by reperfusion of the ischaemic myocardium. J Mol Cell Cardiol 14: 483-485, 1982.

DECATERINA R, GIANNESSI D, MAZZONE A, BERNINI W, LAZZERINI G, MAFFEI S, CERRI M, SALVATORE L, WEKSLER B: Vascular prostacyclin is increased in patients ingesting omega-3 polyunsaturated fatty acids before coronary artery bypass graft surgery. Circulation 82: 428-438, 1990.

EPSTEIN AE, DIMARCO JP, ELLENBOGEN KA, ESTES NA 3rd, FREEDMAN RA ET AL.: ACC/AHA/HRS 2008 Guidelines for Device-Based Therapy of Cardiac Rhythm Abnormalities: a report of the American College of Cardiology/American Heart Association Task Force on Practice Guidelines. Circulation 117: e350-e408, 2008.

GAO F, TAHA AY, MA K, CHANG L, KIESEWETTER D, RAPOPORT SI: Aging decreases rate of docosahexaenoic acid synthesis-secretion from circulating unesterified $\alpha$-linolenic acid by rat liver. AGE (Dordr) 35: 597-608, 2013.

HARRIS WS, VON SCHACKY C: The omega-3 index: a new risk factor for death from coronary heart disease? Prev Med 39: 212-220, 2004.

JIANG H, QUILLEY J, DOUMAD AB, ZHU AG, FALCK JR, HAMMOCK BD, STIER CT JR, CARROLL MA: Increases in plasma trans-EETs and blood pressure reduction in spontaneously hypertensive rats. Am J Physiol 300: H1990-H1996, 2011.

LIN WK, FALCK JR, WONG PY: Effect of 14,15-epoxyeicosatrienoic acid infusion on blood pressure in normal and hypertensive rats. Biochem Biophys Res Commun 167: 977-981, 1990.

LONDON B, ALBERT C, ANDERSON ME, GILES WR, VAN WAGONER DR, BALK E, BILLMAN GE, CHUNG M, LANDS W, LEAF A, MCANULTY J, MARTENS JR, COSTELLO RB, LATHROP DA: Omega-3 fatty acids and cardiac arrhythmias: prior studies and recommendations for future research: a report from the National Heart, Lung, and Blood Institute and Office of Dietary Supplements Omega-3 Fatty Acids and Their Role in Cardiac Arrhythmogenesis Workshop. Circulation 116: e320-e335, 2007.

MITASIKOVA M, SMIDOVA S, MACSALIOVA A, KNEZL V, DLUGOSOVA K, OKRUHLICOVA L, WEISMANN P, TRIBULOVA N: Aged male and female spontaneously hypertensive rats benefit from n-3 polyunsaturated fatty acids supplementation. Physiol Res 57 (Suppl 2): S39-S48, 2008.

NAIR SS, LEITCH JW, FALCONER J, GARG ML: Prevention of cardiac arrhythmia by dietary (n-3) polyunsaturated fatty acids and their mechanism of action. J Nutr 127: 383-393, 1997.

PEARSON T, WARREN AY, BARRETT DA, KHAN RN: Detection of EETs and HETE-generating cytochrome P450 enzymes and the effects of their metabolites on myometrial and vascular function. Am J Physiol 297: E647-E656, 2009.

RADOSINSKA J, BACOVA B, BERNATOVA I, NAVAROVA J, ZHUKOVSKA A, SHYSH A, OKRUHLICOVA L, TRIBULOVA N: Myocardial NOS activity and connexin-43 expression in untreated and omega-3 fatty acids-treated spontaneously hypertensive and hereditary hypertriglyceridemic rats. Mol Cell Biochem 347: 163-173, 2011.

RADOSINSKA J, BACOVA B, KNEZL V, BENOVA T, ZURMANOVA J, SOUKUP T, ARNOSTOVA P, SLEZAK J, GONÇALVESOVA E, TRIBULOVA N: Dietary omega-3 fatty acids attenuate myocardial arrhythmogenic factors and propensity of the heart to lethal arrhythmias in a rodent model of human essential hypertension. J Hypertens 31: 1876-1885, 2013.

RUPP H, RUPP TP, ALTER P, MAISCH B: Mechanisms involved in the differential reduction of omega-3 and omega6 highly unsaturated fatty acids by structural heart disease resulting in "HUFA deficiency". Can J Physiol Pharmacol 90: 55-73, 2012.

SUKRUTHA SK, ADAMECHOVA Z, KUNDER RB, SAVITHA J, CERTIK M: Endophytic fungi: Are they potential candidates for the production of polyunsaturated fatty acids? Int J Appl Microbiol Sci 1: 44-49, 2012.

TAHA AY, BASSELIN M, RAMADAN E, MODI HR, RAPOPORT SI, CHEON Y: Altered lipid concentrations of liver, heart and plasma but not brain in HIV-1 transgenic rats. PLEFA 87: 91-101, 2012.

TRANCHIDA F, TCHIAKPE L, RAKOTONIAINA Z, DEYRIS V, RAVION O, HIOL A: Long-term high fructose and saturated fat diet affects plasma fatty acid profile in rats. J Zhejiang Univ Sci B 13: 307-317, 2012. 
TRIBULOVA N, SEKI S, RADOSINSKA J, KAPLAN P, BABUSIKOVA E, KNEZL V, MOCHIZUKI S: Myocardial $\mathrm{Ca}^{2+}$ handling and cell-to-cell coupling, key factors in prevention of sudden cardiac death. Can J Physiol Pharmacol 87: 1120-1129, 2009.

VIVIANI ANSELMI C, FERRERI C, NOVELLI V, RONCARATI R, BRONZINI R, MARCHESE G, SOMALVICO F, CONDORELLI G, MONTENERO AS, PUCA AA: Fatty acid percentage in erythrocyte membranes of atrial flutter/fibrillation patients and controls. J Interv Card Electrophysiol 27: 95-99, 2010.

VON SCHACKY C: Omega-3 Index and sudden cardiac death. Nutrients 2: 375-388, 2010.

VON SCHACKY C, HARRIS WS: Cardiovascular risk and the omega-3 index. J Cardiovasc Med 8 (Suppl 1): S46S49, 2007.

YOUSIF MH, BENTER IF: Role of 20-hydroxyeicosatetraenoic and epoxyeicosatrienoic acids in the regulation of vascular function in a model of hypertension and endothelial dysfunction. Pharmacology 86: 149-156, 2010.

ZEGHICHI-HAMRI S, DE LORGERIL M, SALEN P, CHIBANE M, DE LEIRIS J, BOUCHER F, LAPORTE F: Protective effect of dietary n-3 polyunsaturated fatty acids on myocardial resistance to ischemia-reperfusion injury in rats. Nutr Res 30: 849-857, 2010.

ZIPES DP, CAMM AJ, BORGGREFE M, BUXTON AE, CHAITMAN B ET AL.: ACC/AHA/ESC 2006 Guidelines for Management of Patients With Ventricular Arrhythmias and the Prevention of Sudden Cardiac Death: a report of the American College of Cardiology/American Heart Association Task Force and the European Society of Cardiology Committee for Practice Guidelines. Circulation 114: e385-e484, 2006. 